\title{
A Mobile-Based High Sensitivity On-Field Organophosphorus Compounds Detecting System for IoT-Based Food Safety Tracking
}

\author{
Han Jin, ${ }^{1,2}$ Yajie Qin, ${ }^{1,2}$ Hao Liang, ${ }^{1}$ Lei Wan, ${ }^{2}$ Hao Lan, ${ }^{3}$ Guoping Chen, \\ Ran Liu, ${ }^{1}$ Li-rong Zheng, ${ }^{1,4}$ Patrick Chiang, ${ }^{2}$ and Zhi-liang Hong ${ }^{2}$ \\ ${ }^{1}$ Center of Micro-Nano Systems, Fudan University, Shanghai 200433, China \\ ${ }^{2}$ State Key Laboratory of ASIC \& Systems, Fudan University, Shanghai 201203, China \\ ${ }^{3}$ School of Electronic Engineering, Xidian University, Xian 710126, China \\ ${ }^{4}$ IME, ICT, KTH Royal Institute of Technology, Stockholm, Sweden \\ Correspondence should be addressed to Yajie Qin; yajieqin@fudan.edu.cn and Guoping Chen; gpchenapple@fudan.edu.cn
}

Received 4 October 2016; Accepted 13 December 2016; Published 20 February 2017

Academic Editor: Xiongchuan Huang

Copyright (C) 2017 Han Jin et al. This is an open access article distributed under the Creative Commons Attribution License, which permits unrestricted use, distribution, and reproduction in any medium, provided the original work is properly cited.

\begin{abstract}
A mobile-based high sensitivity absorptiometer is presented to detect organophosphorus (OP) compounds for Internet-of-Things based food safety tracking. This instrument consists of a customized sensor front-end chip, LED-based light source, low power wireless link, and coin battery, along with a sample holder packaged in a recycled format. The sensor front-end integrates optical sensor, capacitive transimpedance amplifier, and a folded-reference pulse width modulator in a single chip fabricated in a $0.18 \mu \mathrm{m}$ 1-poly 5-metal CMOS process and has input optical power dynamic range of $71 \mathrm{~dB}$, sensitivity of $3.6 \mathrm{nW} / \mathrm{cm}^{2}(0.77 \mathrm{pA})$, and power consumption of $14.5 \mu \mathrm{W}$. Enabled by this high sensitivity sensor front-end chip, the proposed absorptiometer has a small size of $96 \mathrm{~cm}^{3}$, with features including on-field detection and wireless communication with a mobile. OP compound detection experiments of the handheld system demonstrate a limit of detection (LOD) of $0.4 \mu \mathrm{mol} / \mathrm{L}$, comparable to that of a commercial spectrophotometer. Meanwhile, an android-based application (APP) is presented which makes the absorptiometer access to the Internet-of-Things (IoT).
\end{abstract}

\section{Introduction}

In recent years, food safety is becoming one of the most concerned social issues. There are various factors threatening food safety during the whole food processing procedures. For example, organophosphorus (OP) pesticides residues have caused serious pollution to food and environment with extensive and sometimes improper application. With the rapid development of wireless communication technologies and intelligent devices, more and more Internet-of-Things (IoT) systems are applied in the food safety tracking field. However, the status information of food can merely be obtained by reading prestoring data in radio frequency identification (RFID) tag, which is inapplicable for on-field detection. Therefore, a fast, on-field, and low cost OP detection system is crucial for food safety.
Numerous analytical reports have been published in the past decades on the OP determination. They can be generally divided into two categories, nonenzymes and enzyme-based methods [1]. The former methods mainly rely on physical and chemical ways without biological components involved, such as gas chromatography [2-6], nitrogen-phosphorus detection $[7,8]$, high-performance liquid chromatography [9-11], and capillary electrophoresis [12]. Normally, these methods heavily depend on fussy instruments and are more suitable for in-laboratory detection. The latter are called enzyme-based biosensors. A biosensor is composed of biological molecules and transducers. Due to the specificity of biological components for the analyte, the biosensors show more and more potential in on-field monitoring. Two types of enzyme are involved in such biosensors: OP hydrolase that can directly hydrolyze OP pesticides [13-15] and acetyl 
cholinesterase (AChE) that can be inhibited by OP pesticides [16]. The analytical method using AChE needs addition of special substrate and detects the inhabitation rate after its exposure to OP, thus resulting in irreversible loss in enzyme activity [17]. Therefore, OP hydrolase based biosensor is applied in the detection system proposed in this paper.

As for biosensor applied in on-field detection, sensitivity is one of the most important characteristics. Generally biosensor consists of biological recognition element and transducer [18], both of which have significant effect on sensitivity. When employing enzyme as the biological recognition element, it is essential to choose suitable immobilization method as it would affect the sensitivity and stability. Typically physical adsorption method is used for immobilization. With the potential for anti-interference and miniaturization [19], optical sensors now are widely employed as transducers. Optical sensors can be divided into two categories according to the light source, fluorescencebased sensors [20] and absorbance-based sensors [19]. The former one can detect OP concentration by measuring fluorescence emission under excitation. However, the fluorescence intensity is heavily depending on $\mathrm{pH}$ change, which limits the sensitivity and response speed. The latter one is to detect OP concentration by measuring transmission light intensity after being absorbed by the solution. Due to the simple principle and structure, it promises high sensitivity and flexibility for practical application. Besides the measurement principle, photosensitive devices and circuits are another key factor that decides the performance of optical sensor. Charge-coupled device (CCD) is usually used as the photodetection sensor in commercial system to achieve high sensitivity [21]. Nevertheless, CCD is not compatible with complementary metal oxide semiconductor (CMOS) process and the photocurrent readout circuit cannot integrate with it. Therefore, the detection system based on CCD device always has higher cost and shows a lower integrated level. Customized phototransistor has also been used as the senor component to improve sensitivity, which has limited dynamic range of measurement yet [22]. With use of special process, specific high-performance sensing components are hard to manufacture and integrate with other circuits in system. In spite of difficulty in improving sensing components, readout circuits can be also optimized to achieve high sensitivity. To improve the sensitivity of the systems, noise of the circuit needs to be reduced including dark current, thermal noise, flick noise, and reset noise. Therefore, different circuit architectures have been proposed to cut down the noise of circuit. In order to lower the dark current of photodiode, a pseudo differential photocurrent readout circuit is proposed which is constituted by two channels [22] to detect dark current and total current, respectively. Techniques such as capacitive control, bandwidth control, and charge control [23] are used to reduce reset noise. Furthermore, $\Delta \Sigma$ modulator and digital filter are also utilized to process the output of capacitive transimpedance amplifier (CTIA) [24] to achieve less noise.

Besides of high sensitivity biosensors, it is essential to design a portable and intelligent OP detection platform for on-field detection. Several schematic designs of portable detection system have been proposed in last decade.

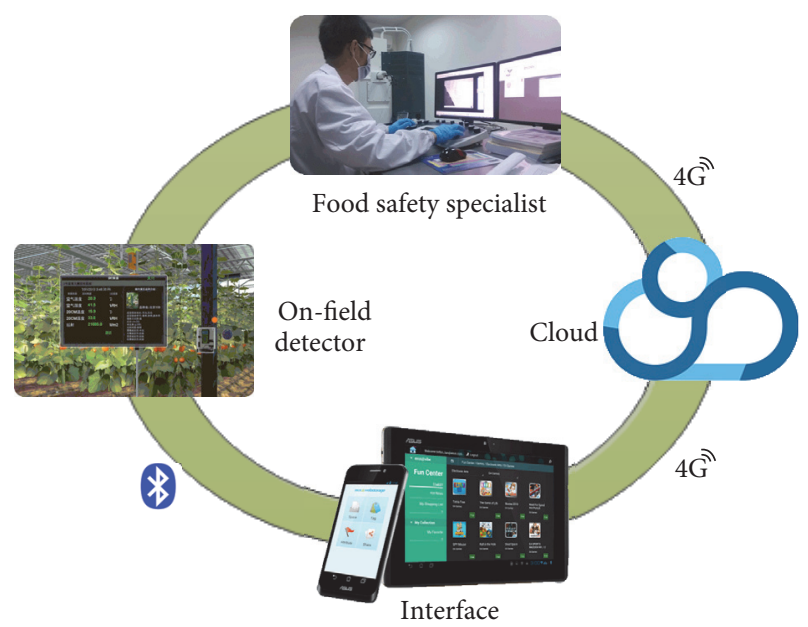

FIgURE 1: Overall view of the OP on-field detection platform based on IoT.

According to the paper-based detection sensor proposed by Kavruk et al. [25], paper containing enzyme mixture is fixed on a thin plastic support to recognize OP compounds, which enhances portability observably. Nevertheless, the detection still needs to be done by measuring color intensity on additional instruments, which is inapplicable for on-field detection. The other approach is to fabricate the detection system by assembling light source, fibers, optical sensors, and other discrete components together [26]. Based on this idea, Choi et al. [26] employ microcontroller and printed circuit board (PCB) to integrate discrete components, which miniaturizes system size and enhances hardware reliability. Besides hardware integration, computer is also employed to control detection process [27], receive and process data through data interface, and display detection result. By involving computer into the detection system, it is promised to improve the usability and intelligence of detection system, as well as miniaturizing terminal size. However, limited by the physical size of computer and effective range of network, it is inapplicable to use such a system outdoors. Fortunately with the rapid development of smart phone and wireless communication technology, it is possible to further improve the system in portability and usability. Figure 1 illustrates the overview of an OP on-field detection based on IoT platform. In this paper, main attention is focused on the implementation of a practical, portable, and high sensitivity OP detection system based on a customized sensor, wireless communication technology, and smartphone application.

The OP detection system proposed in this work demonstrates several improvements in the performance of customized front-end sensor and the minimization of system compared with previously reported system. In order to achieve both high sensitivity and large dynamic range at the same time, a sensor front-end with folded-reference is proposed and employed. Meanwhile, photodiode is manufactured in conventional CMOS process and integrated in sensor front-end chip, helpful for minimization of system. Considering the requirement of handheld system, the system 


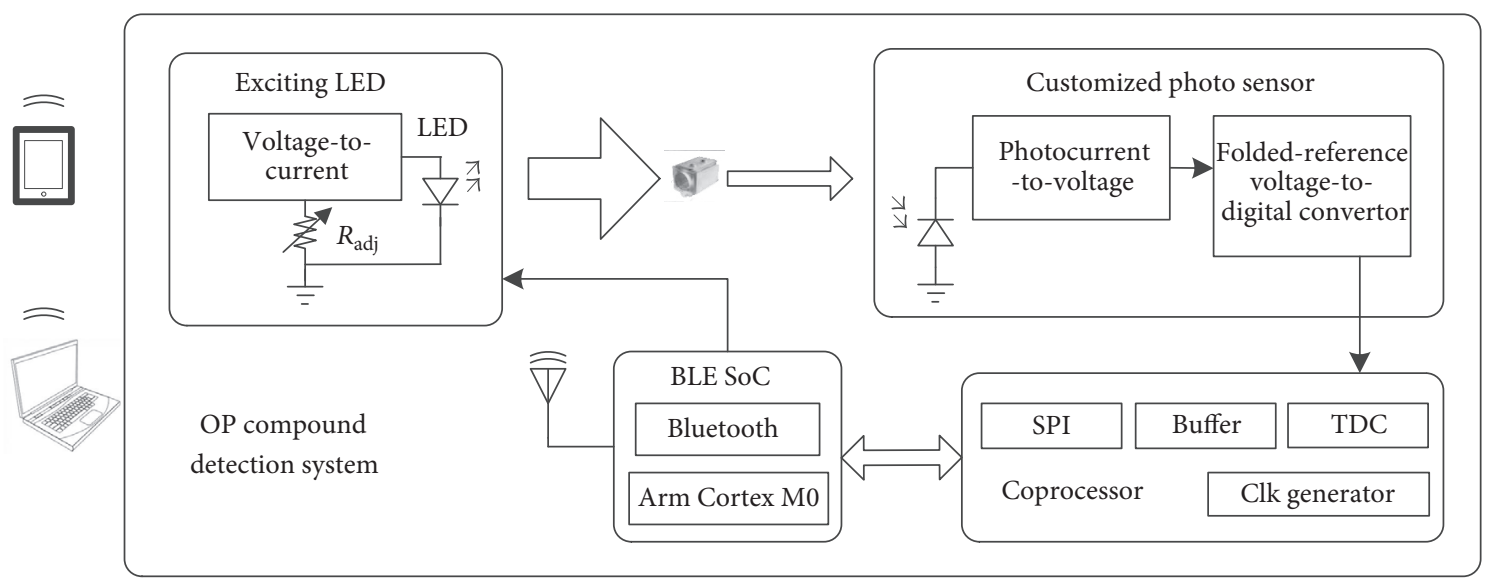

FIGURE 2: The schematic of proposed handheld organ phosphorus compounds detection system.

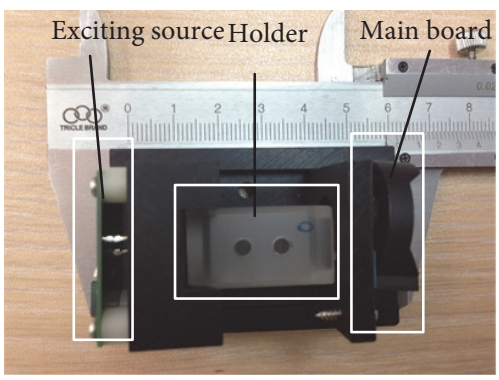

(a)

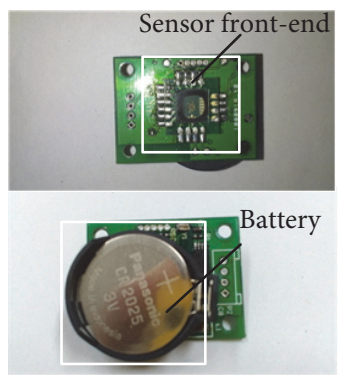

(b)

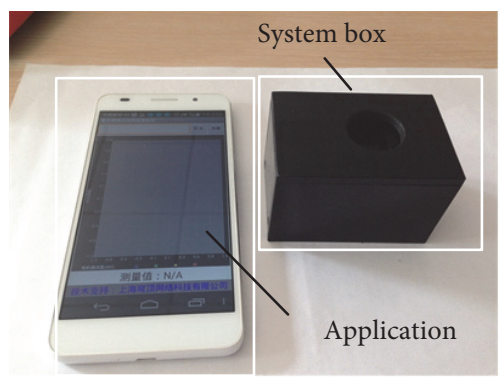

(c)

FIGURE 3: (a) Internal structure of the system. (b) The main board of the system. (c) The photograph of OP compound detection system.

box is designed to be small size and light weight for high performance in portability and mobility. By employing Bluetooth protocol and smartphone application, detection data can be wirelessly transmitted to smartphone, then further processed, and displayed on smartphone, which reduces power consumption of the detection part. Besides, benefiting from the high sensitivity of photodetection sensor, only trace solution is enough to get high accuracy; hence, very little enzyme is utilized. All of these features mentioned above promise the proposed system to be portable with high sensitivity and low cost.

This paper is organized as follows. In Section 2, the whole system and details of each block are introduced. In Section 3, the working principle and the noise analysis of the photodetection sensor are described. Section 4 shows measurement results of the photodetection sensor and OP compounds detection and Section 5 concludes this paper.

\section{Principle and Implementation of Detection System}

The biosensing principle mainly detects the absorbance of the enzymatic product hydrolyzed from methyl parathion. The diagram of the proposed absorptiometer is illustrated in Figure 2, which is mainly composed of a meter-box and an application software (APP) running in an android-based smart mobile. The performance of the system is determined by the compact optical path. In the meter-box, a narrowband light emitting diode (LED) is used as the light source, where intensity is programmed by the on-board microcontroller unit (MCU) through an embedded digital-to-analog converter $(\mathrm{ADC})$ and a voltage-to-current converter. The light after passing through the sample holder is detected by the customized sensor front-end and converted into voltage pulse width modulated (PWM) signals, which represent the information with their pulse width. The converted voltage pulse signals are further quantized by the on-board MCU with its intrinsic timer. The meter-box communicates with the paired smart mobile through a Bluetooth link. APP operating in the smart mobile is used for controlling the detection flow, data analysis, and results figuring.

2.1. Meter-Box Implementation. The electronic system of the meter-box is implemented in two boards, as shown in Figure 3(a): the exciting source board and the main board. The exciting source board integrates narrowband LED, which fulfills voltage-to-current conversion in a rather simple way to power the LED. The main board shown in Figure 3(b) 
integrates the sensor front-end chip [28] as well as the necessary current bias and voltage reference circuits, while Coprocessor, Bluetooth low energy (BLE), system on chip (SoC), and power management integrated circuits (ICs) are also arranged on the other side of main board, which communicates with the mobile phone, provides an adjustable voltage reference to exciting source board to tune the light intensity and its on/off, and powers the sensor chip as well as quantizing the PWM signal received from the sensor chip.

The sample holder is made of quartz glass and can be used to store the detected solution, as depicted in Figure 3(a). The holder is hermitical except for two circular holes on top for injection of solutions, such as OP compounds, OP hydrolase, and buffer solution. For the handheld system is designed to use in the field environment and easily measure the OP compounds, the OP compounds hydrolase is fixed on the inner wall of the quartz glass by absorption and the sample is hydrolyzed in the holder. The light from the LED on the exciting source board transmits through sample holder to sensing detector on the main board. The collected light should completely be light modulated in theory by the monitored solution without the unmodulated light, which can cause measurement error. The unmodulated light mainly comes from two sources, external leaked light leakage and multi-internal reflecting light caused by inside wall. In order to reduce external leaked light, a highly sealed box is built to wrap up the whole system, showed in Figure 3(c). On the other hand, light-absorbing material is coated on the inner wall of the box to lower the multi-internal light reflection. What is more, the groove in the box is used to fix the sample holder and make sure the optical path is the same in each test, which is essential for the consistency of measurement results.

In this work, a blue $(400 \mathrm{~nm})$ LED is used. Due to its huge power consumption, the blue LED is turned on before each test and turned off after the test, controlled by the BLE SoC to reduce the power consumption. A sample command is send out from the smartphone through Bluetooth module; Arm Cortex M0 turns on the LED. Secondly, Coprocessor digitalizes the output of the light detection sensor and computes the intensity of the light passing through the undertesting sample liquid. Thirdly, after getting the concentration, MCU sends the data to smartphone and turns off the LED. The specific APP running the android-based smartphone computes out the absorbance and the concentration of the sample and reports the results. Hence, a period of the measuring is completed.

The size of the meter-box is only $96 \mathrm{~cm}^{3}$. Because of the smaller volume and less weight, mobility and portability of system have been improved significantly, much more suitable for field environment application. Only $3.6 \mathrm{~mL}$ of tested solution is needed to achieve sufficient accuracy with the help of noise reduction operation and shorter optical path. In this way, every test costs less hydrolytic enzyme.

2.2. Mobile APP and Operation Flow. The complete OP compounds detection can be divided into two phases: calibrating phase and measuring phase. In calibrating phase, two different fixed concentrations of solution are sent to the handed system to extract the fitting function and coefficients.
The function of the fitting line is

$$
\begin{aligned}
& y=k \cdot \ln \xi+b \\
& k=\frac{y_{1}-y_{2}}{\ln \xi_{1}-\ln \xi_{2}} \\
& b=y_{1}-k \cdot \ln \xi_{1},
\end{aligned}
$$

where $k, b$ are the slop and the liner intercept of the fitting line and $\xi$ is the light intensity output of the photodetection sensor. Another important parameter in OP compounds detection, the limit of detection (LOD) is also extracted in this calibration phase. The expressing of LOD is

$$
\mathrm{LOD}=\frac{3 \cdot \sigma}{k},
$$

where $\sigma$ is the standard deviation with the $0 \mathrm{uM}$ concentration solution.

To extend the battery life of the meter-box, microcod/APP codesign method is used. The detailed operation flow is shown in Figure 4. The first step of operation is to use APP to send sample start command to BLE SoC. After the BLE SoC receives interpret ask, it sends "sampling start" command to Coprocessor through SPI interface. Before acknowledging interpret ask, Coprocessor is turned down, which takes a majority of power consumption during sampling operation. Receiving interpret response, Coprocessor powers up and starts to conduct sampling command. There are 3 steps for Coprocessor to do during sampling. First, it realizes self-configuration. Secondly, it sends divided clock signal to drive photo sensor. Finally, Coprocessor decodes PMW signal received from sensor and stores converted data in buffer. After getting enough data, the Coprocessor sends interpret ask signal and sampling results to BLE SoC through SPI interface before powering down. Then BLE SoC stores data in local buffer until APP sends data read command. When APP gets data from BLE SoC, the final result is calculated by specific principle with data received. The data processing includes absorbance calculation, LOD calculation, and linearity analysis and result plot. During communication, generic attribute (GATT) protocol is implemented to avoid possible error caused by overflow and empty. In addition, LED and Coprocessor, costing most power, are turned down during free period, which realizes lower power strategy.

\section{Photodetection Sensor Architecture}

The main function of the sensor is converting the light intensity to digital signal. In general, a photodetection sensor is usually consisted of photosensitive component, current readout circuit, and quantization circuit. The photosensitive component converts the light intensity to photo current and the current readout circuit converts the current to voltage. Quantization circuit converts the voltage to digital signal. Charge-coupled device (CCD) and photodiode fabricated by CMOS process can be used as photosensitive component. CCD has a higher sensitivity than CMOS photodiode. However, CCD cannot fabricate with the readout circuit since CCD is not compatible with the CMOS process. There 

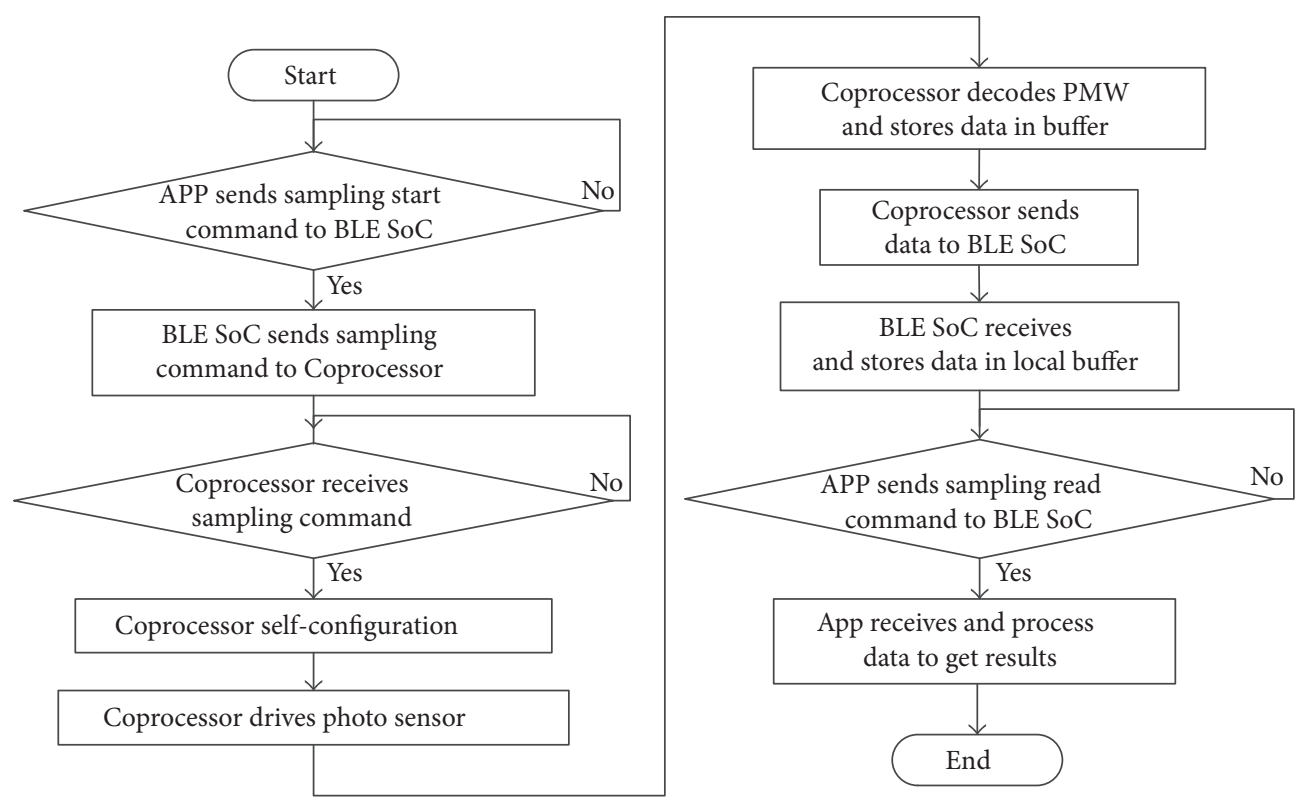

FIgure 4: Operation flow.

are three different types of photodiode in standard CMOS process including $\mathrm{n}+/ \mathrm{p}$-sub, $\mathrm{n}$-well $/ \mathrm{p}$-sub, and $\mathrm{p}+/ \mathrm{n}$-well $/ \mathrm{p}$ sub. In this work, the photodiode is designed with $n$-well/psub. In terms of sensitivity, this type of photodiode performs best because of it has wider depletion region and larger sidewalls. The signal-to-noise ratio might be high than $\mathrm{n}+\mathrm{p}$ sub, $\mathrm{p}+/ \mathrm{n}$-well $/ \mathrm{p}$-sub by a factor of 1.5 and 1.2 , respectively [30].

3.1. Architecture of Detection Sensor. The photo current can be converted into voltage by integration through capacitor or resistor. Capacitive feedback integration needs periodic reset to address the saturation problem, while the resistive feedback does not. However, capacity feedback integration is less noisy than the resistive feedback. Hence, the capacitive feedback integration is used in this work.

Previous works in CMOS typically utilize an ADC to quantize the output voltage of the TIA [31], resulting in several disadvantages. For example, a capacitive successive approximation register (SAR) ADC can be low power but has large area overhead. On the other hand, a sigma-delta ADC can achieve better resolution but consumes significant power. In this work, the voltage signal is converted into a pulse signal for reduced complexity and lower power consumption.

The architecture of the photodetection sensor and the interface with MCU are depicted in Figure 5. The photodetection sensor is consisted of a TIA with a T-type lowleakage current switch, two comparators, a ramp generator, and several logic circuits. For the input photocurrent is about a few pA $\left(10^{-12} \mathrm{~A}\right)$, the $T$-type switch is essential to reduce the current leakage. The capacitor feedback transimpedance amplifier (CTIA) is used to convert the photocurrent to voltage. The sequential comparators and the logical circuit are used to convert the voltage signal to pulse width modulated signal (PWM). Comparator CMP1 with fixed reference voltage $V_{\text {ref2 }}$ generates the positive edge of pulse wave, while CMP2 with folded-reference Vref1 provides the negative edge. A MCU is used to provide the clock signal to control the integration time and compute the pulse wave of the output logical control circuit. One fixed voltage reference and a folded reference instead of two fixed references are employed in here to enlarge the dynamic range of the input light intensity.

3.2. Operating Principle. One major problem for conventional PWM-based optical detection systems is that when the received input optical power is too small, the detector takes excessive time to perform quantization. In order to address this problem, this work utilizes a folded-reference voltage rather than a fixed reference in order to decrease integration time. Specifically, during $0 \sim T_{1}, V_{\text {ref } 1}$ is a fixed voltage, similar to a conventional PWM-based system. When the output voltage of the TIA is larger (less) than $V_{1}$ at $T_{1}$, $V_{\text {ref1 } 1}$ is a fixed voltage (ramp signal) during $T_{1} \sim T_{2}$. Figure 6 describes these two conditions mentioned above.

When $V_{\text {ref1 } 1}$ is a folded-reference, the expression is as follows, with (3). The output voltage of the TIA across the two reference voltages $V_{\text {ref2 }}$ and $V_{\text {ref1 }}$ at times $t_{r 1}$ and $t_{r 2}$, respectively, is turned into a PWM signal by two comparators and proceeding logical gates. The photocurrent can be expressed as (4), where $C_{f}$ is the integration capacitor. The integration time $T_{\mathrm{pi}}$ required to cross the two different voltages is expressed in (5).

$$
V_{\text {ref1 }}= \begin{cases}V_{1} & \left(0<t_{r 2} \leq T_{1}\right) \\ \left(t_{r 2}-T_{1}\right) \cdot \frac{V_{\mathrm{CM}}-V_{1}}{T_{2}-T_{1}}+V_{1} & \left(T_{1}<t_{r 2} \leq T_{2}\right)\end{cases}
$$




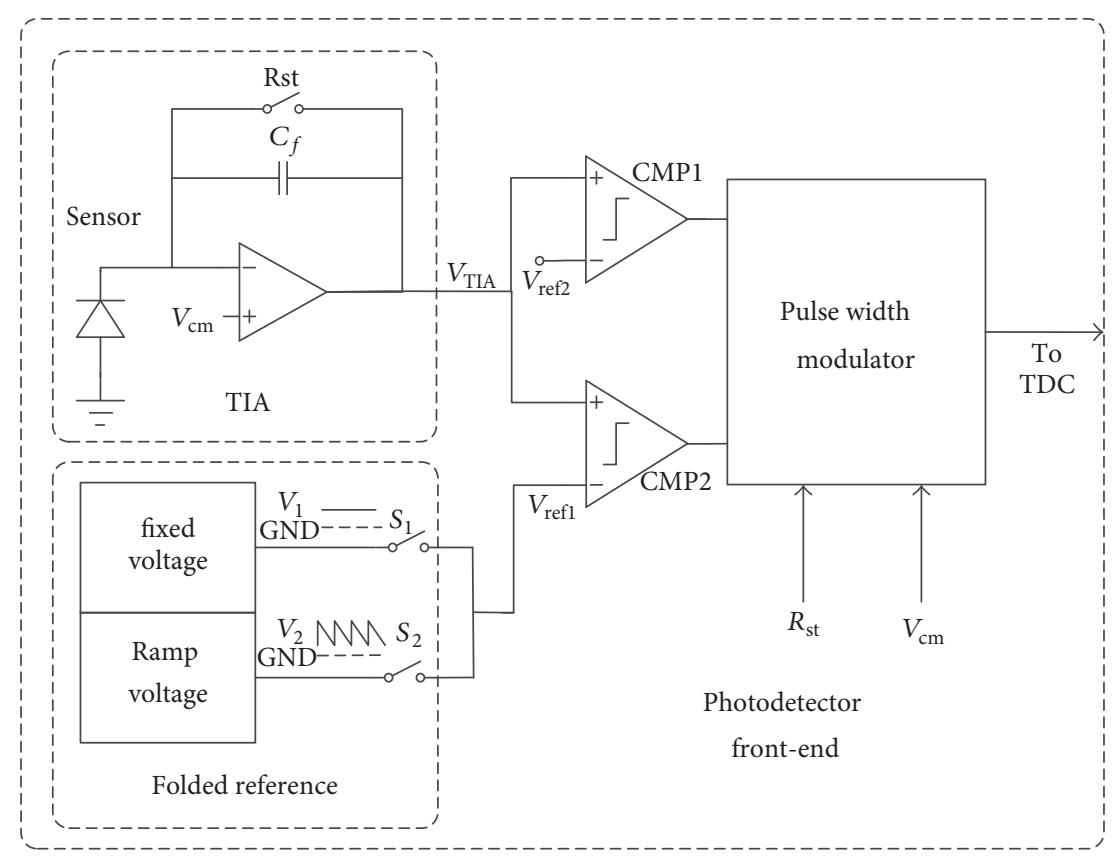

FIGURE 5: Architecture of the folded-reference detector.

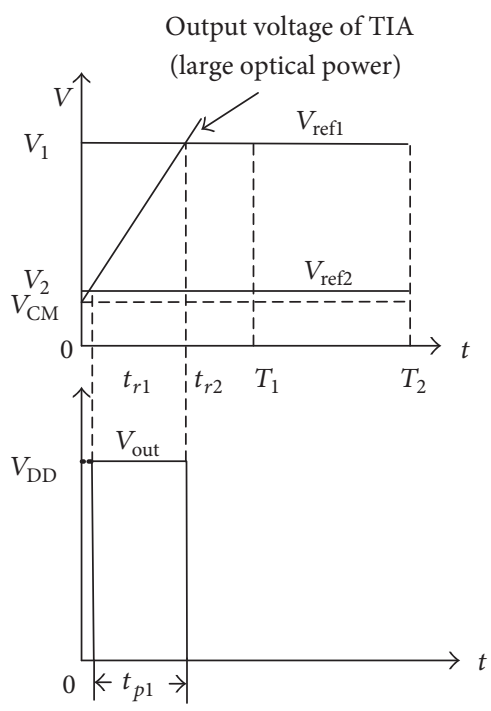

(a)

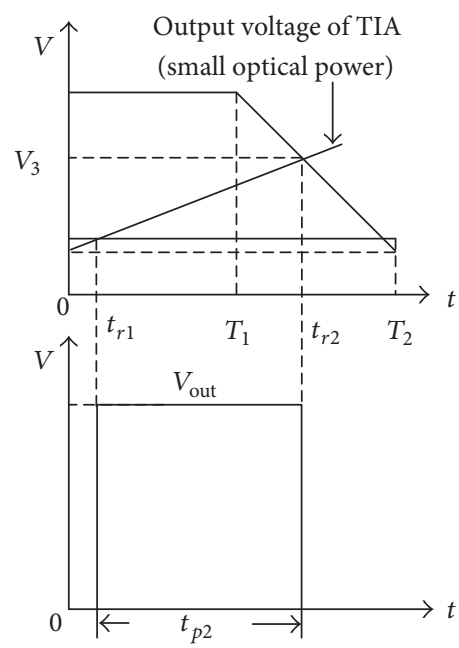

(b)

FIGURE 6: Operating principle of the folded-reference detector when input optical power is (a) large or (b) small.

$I_{\text {photo }}=C_{f} \cdot \frac{\left(V_{\text {ref } 1}-V_{\text {ref2 }}\right)}{\left(t_{r 2}-t_{r 1}\right)}$

$T_{\text {pi }}=\left(V_{\text {ref } 1}-V_{\text {ref } 2}\right) \cdot \frac{C_{f}}{I_{\text {photo }}}$.

In case $V_{\text {ref1 }}$ is a fixed voltage when the photocurrent $I_{\text {photo }}$ is small, the difference between $V_{\text {ref1 }}$ and $V_{\text {ref2 }}$ is quite large, and $T_{\mathrm{pi}}$ can be very large. However, if a folded reference is adopted, such as in expression (4), the integration time $T_{\mathrm{pi}}$ can be significantly reduced. The integration times for conventional fixed reference and the proposed folded reference have been simulated, which are different. As can be seen in Figure 7, when the photocurrent is lower than $10 \mathrm{pA}$, the integration time of the proposed reference ascends very slowly compared with the fixed reference.

3.3. Capacitive Feedback Transimpedance Amplifier (CTIA). The CTIA illustrated in Figure 8 consists of a feedback capacitor, an OTA, and a low-leakage switch. In general, the traditional switch has a leakage current of several pA [32] due to the subthreshold current. Considering that the 


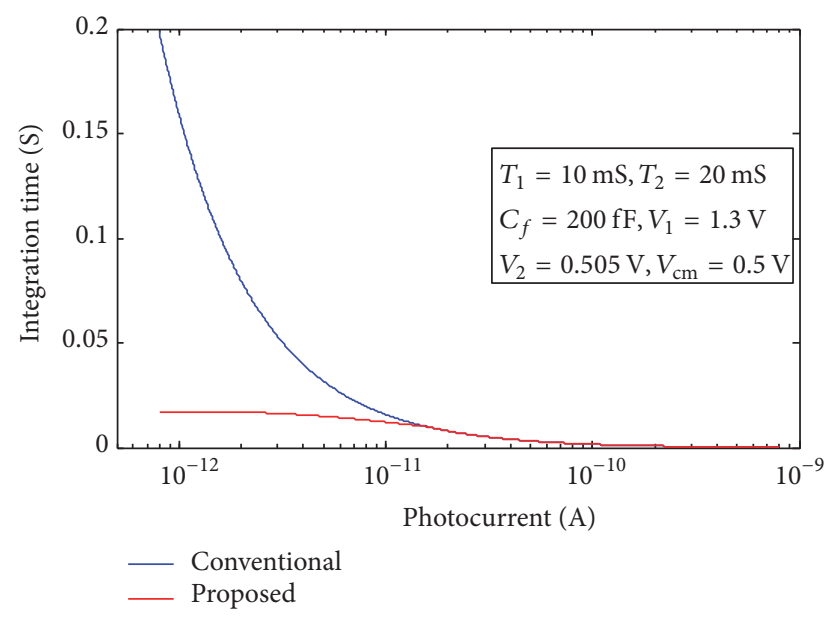

Figure 7: Photocurrent versus integration time.

photocurrent is about a few $\mathrm{pA}$, the traditional switch is not suitable used in this work. The leakage of the transistor can be expressed as follows:

$$
I_{D S} \approx I_{D 0} \frac{W}{L} e^{V_{G S} / n V_{T}}\left(1-e^{-V_{D S} / V_{T}}\right),
$$

where $I_{D S}$ and $I_{D 0}$ are the leakage current of the transistor and the saturate current successively, $W$ and $L$ are the width and the length of the transistor, and $n$ is a process related parameter. To minimize the leakage current, a $T$-type switch [33] is utilized.

3.4. Noise Analysis. There are many kinds of noise in the photodetection sensor including shot and $1 / f$ noise in the photodiode, thermal, and $1 / f$ noise in the CTIA and the two comparators, charge injections in the switch. The correlated double sampling (CDS) adopted in the sensor reduces noise of charge injection. The operation amplifier needs to be designed carefully to reduce the thermal and $1 / f$ noise. The n-well/p-subphotodiode has high noise compared to the $n+/ p$-sub and $p+/ n$-well/p-sub [34], possibly due to the larger photocurrent than the other photodiode. The hysteresis comparator is adopted in the circuit to reduce the jitter when the output voltage is converted to pulse wave. After the pulse wave is digitalized by the counter in MCU, the data is sent to a digital filter to get a better performance. In the circuit, parameters such as feedback capacitor and integration time need to be selected to meet noise and current-to-voltage gain requirement.

Noise of integrator and photodiode is

$$
\begin{aligned}
d I_{D}^{2} & =2 q I_{D} d f \\
d I_{D f}^{2} & =I_{D} \frac{K F_{D}}{A_{D}} \frac{d f}{f} d f .
\end{aligned}
$$

There are two main kinds of noise, shot noise $d I_{D}{ }^{2}$ and the $1 / f$ noise $d I_{D f}^{2}$ in the photodiode [35].

$$
e_{M}{ }^{2} \approx 4 k T\left(\frac{2 / 3}{g_{m}}\right) d f+\frac{K F_{F}}{\mathrm{WLC}_{o x}{ }^{2}} \frac{d f}{f} .
$$

Another noise source is the thermal and $1 / f$ noise of the operation amplifier which could be expressed in (8).

Other types of noise in the photodiode include dark current and the thermal noise of the parasitic resistance. It can be expected that the shot noise and the $1 / f$ noise are positive correlation with the photocurrent.

The main noise of integrator is the thermal noise and the $1 / f$ noise. The influence of the charge injection is still necessary to take into consideration. There are two phases in the photodetection including reset phase and the integration phase. For the transmission function of the two phases are different, the noise is analyzed separately.

(1) Reset Phase. During reset phase, the switch is off; the integrator is set to unit gain. The output noise of the integrator can be expressed as follows:

$$
S_{v, r s t}=e_{M}^{2}+e_{v 1}^{2}+e_{v 2}^{2}+e_{\text {diode }}^{2},
$$

where $e_{M}, e_{v 1}$, and $e_{v 2}$ denote the thermal noise and the $1 / f$ noise of the operating amplifier, comparators 1 and 2 in Figure $9, e_{\text {diode }}$ is produced by the photodiode, and the expressing is

$$
e_{\text {diode }}^{2}=\frac{\left(d I_{D}^{2}+d I_{D f}^{2}\right)}{Y_{M}^{2}} .
$$

$Y_{M}$ is the admittance of the photodiode. The input referred current noise during reset is

$$
S_{I, r s t}=S_{v, r s t} \cdot\left(\frac{C_{F}}{T_{\text {int }}}\right)^{2} .
$$

(2) Integrating Phase. During the integrating phase, the output current of the photodiode is integrated in the feedback capacitor $C_{f}$. If the integration time is $T_{\text {int }}$, the output voltage of the integrator is [34]

$$
V_{\text {out }}(t)=\frac{1}{C_{F}} \cdot i_{\mathrm{pc}} \cdot\left(u(t)-u\left(t-T_{\text {int }}\right)\right) .
$$

$i_{\mathrm{pc}}$ is the photocurrent and the $u(t)$ is the unit step signal. To better understand the frequency character of the noise, the Laplace transformation of the integrator in Figure 10 is

$$
\begin{gathered}
L\left(\frac{1}{C_{F}} \cdot i_{\mathrm{pc}}(t) \cdot\left(u(t)-u\left(t-T_{\mathrm{int}}\right)\right)\right) \\
=\frac{1}{C_{F} \cdot S} \cdot i(S) \cdot\left(1-e^{-T_{\mathrm{int}} \cdot S}\right) .
\end{gathered}
$$

The power spectral density (PSD) of the output of the integrator is

$$
\begin{aligned}
S_{V, \text { int }}= & \left(e_{M}{ }^{2}+e_{\text {diode }}{ }^{2}\right) \cdot\left(\frac{Y_{M}}{C_{F} \cdot S} \cdot\left(1-e^{-T_{\text {int }} S}\right)\right)^{2} \\
& +e_{v 1}{ }^{2}+e_{v 2}{ }^{2} .
\end{aligned}
$$




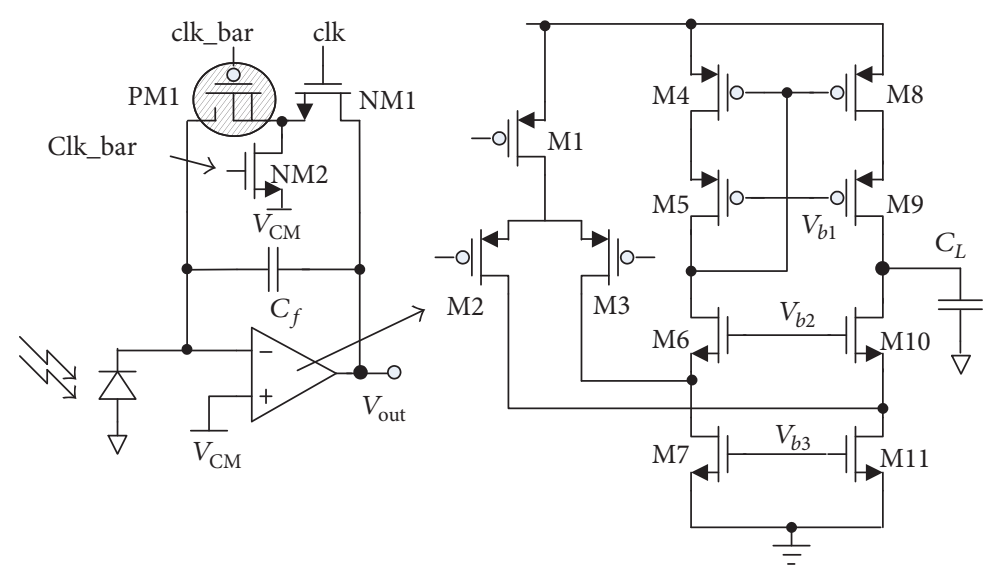

FIGURE 8: CTIA of the photodetection sensor.

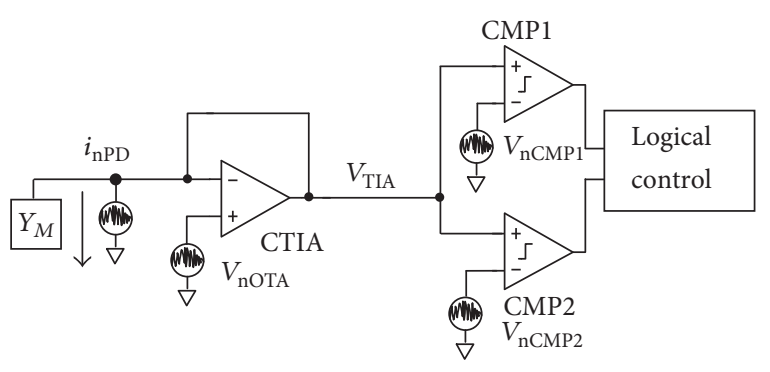

FIGURE 9: Noise model for the photodetection sensor during reset phase.

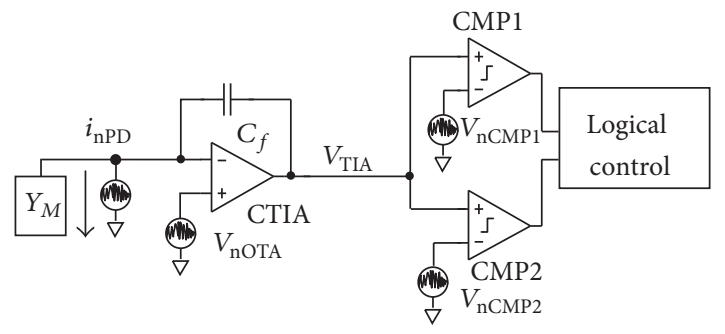

Figure 10: Noise model for the photodetection sensor during integration phase.

From equation above, the input referred current noise is

$$
\begin{aligned}
S_{I, \text { int }}= & S_{V, \text { int }} \cdot\left(\frac{C_{F}}{T_{\text {int }}}\right)^{2} \\
S_{I, \text { int }}= & \left(e_{M}^{2}+e_{\text {diode }}^{2}\right) \cdot\left(\frac{Y_{M}}{T_{\text {int }} \cdot S} \cdot\left(1-e^{-T_{\text {int }} \cdot S}\right)\right)^{2} \\
& +\left(e_{v 1}^{2}+e_{v 2}^{2}\right) \cdot\left(\frac{C_{F}}{T_{\text {int }}}\right)^{2} .
\end{aligned}
$$

The compromise between noises, current-to-voltage gain, sample rate, and sensitivity are taken into consideration. Increasing the area of photodiode can increase the photocurrent.
From the equation above, longer integration time of the CTIA will reduce the input referred current noise, but the sample rate of the photocurrent sensor will reduce either. The parasitic capacitance will increase the input current noise. Increasing the feedback capacitance of the CTIA can improve PSD of the output of integrator. In this work, the integration time is $20 \mathrm{mS}$ and feedback capacitor is $200 \mathrm{fF}$.

\section{Measurement Results}

4.1. Reagent Preparation. The sodium tetraborate (ST, pH 9.18) is got from Shanghai Hongbei Reagent Co., Ltd. (Shanghai, China) and used as buffer solution. Methyl parathion is got from Sigma (St. Louis, MO, USA) and is used directly without purification. Methyl parathion is dissolved in the mixture of water and ethanol and stored at temperature $4^{\circ} \mathrm{C}$ before use.

4.2. Sensor Chip Characteristics. The measurement setup of the chip is shown in Figure 11(a). A tungsten lamp is used as the light source, exhibiting a wide spectral range and high stability in the output optical power. The tungsten lamp is connected to a monochromator that can produce a monolight with adjustable optical power. Then the monolight from the monochromator is sent to the common end of a $Y$-type optical fiber bundles. The $Y$-type optical fiber bundles have two split ends with equal optical power each other. One split end of the $Y$-type optical fiber bundles is connected to a commercial optical power meter (Thorlabs PM100D) for quantizing the input incident power and the other split end of the $Y$-type fiber is connected through an attenuator to the proposed sensor chip. After calibrating the ratio of the outputs of the two optical fibers, the amount of optical power attenuated by the attenuator can be obtained. The clock is generated by a microcontroller (MSP430F149, TI) and the $1.8 \mathrm{~V}$ power supply is generated by a low dropout regulator. The output PWM signal from the proposed sensor chip is sent to a signal acquisition board (PXI-6542, NI) and the data is later postprocessed in MATLAB.

The optical detection sensor chip was implemented in a $0.18 \mu \mathrm{m}$ CMOS process. The power consumption of the 


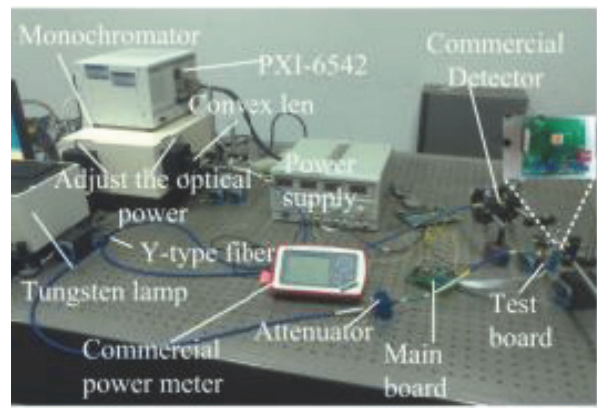

(a)

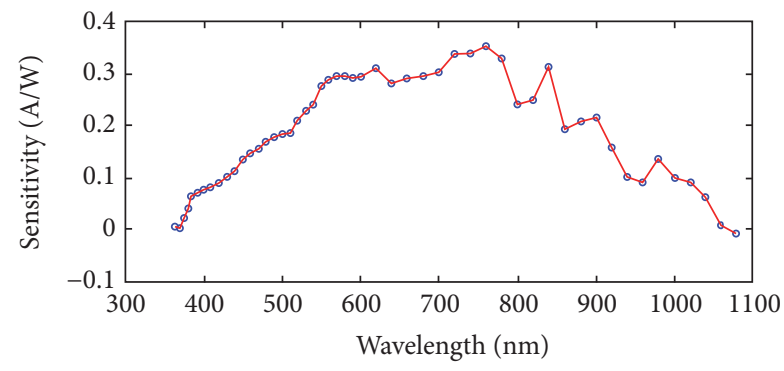

(b)

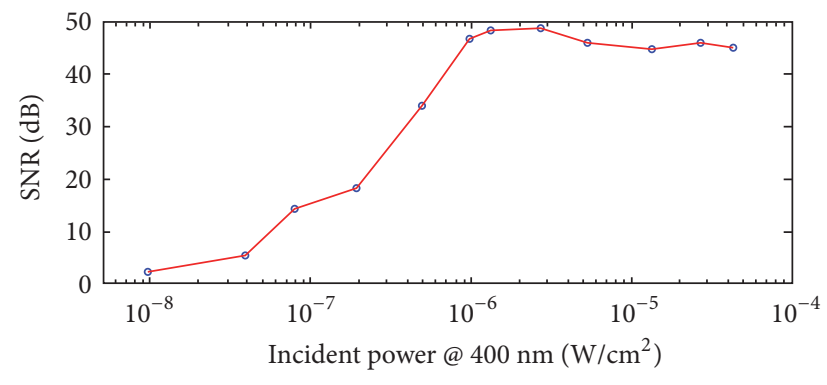

(c)

FIGURE 11: (a) Measurement environment setup. (b) Measured sensitivity of the n-well/p-subphotodiode sensor. (c) SNR of the chip with increasing incident power at $400 \mathrm{~nm}$ and a $20 \mathrm{~ms}$ exposure time.

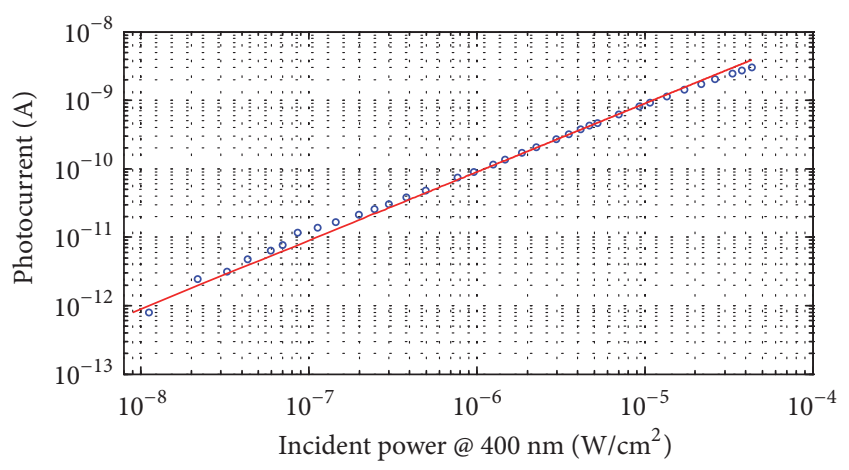

Figure 12: Photocurrent versus incident power at $400 \mathrm{~nm}$ with a 20 ms exposure time.

entire sensor is $14.5 \mu \mathrm{W}$. Because the enzymatic products show the highest absorption at around $400 \mathrm{~nm}$, we choose the monolight for analysis, centered at $400 \mathrm{~nm}$.

The lowest power that can be detected by the chip at a signal-to-noise ratio (SNR) of $0.8 \mathrm{~dB}$ is $3.6 \mathrm{nW} / \mathrm{cm}^{2}$. A total of 25 optical powers were measured from $3.6 \mathrm{nW} / \mathrm{cm}^{2}$ to $13.9 \mu \mathrm{W} / \mathrm{cm}^{2}$, for a total dynamic range of $71.7 \mathrm{~dB}$. The chip was run at a reset frequency of $50 \mathrm{~Hz}$ with an exposure time of $20 \mathrm{~ms}$.

During the measurement of the customized sensor chip, a $10 \mathrm{MHz}$ clock signal in the signal acquisition board was used to digitize the PWM signal of the optical sensor. For integration capacitor of $200 \mathrm{fF}$, the measured photodiode responsivity was $0.075 \mathrm{~A} / \mathrm{W}$ at $400 \mathrm{~nm}$, approximately $21.3 \%$ of the maximum sensitivity at $760 \mathrm{~nm}$.

The out signal without any light stimulus (dark signal) was measured with an integration time of $9.7 \mathrm{~s}$ and an integration capacitor of $200 \mathrm{fF}$. This dark signal is expressed as a ramp from $0.5 \mathrm{~V}$ to $1.3 \mathrm{~V}$ during the integration period. This implies a dark signal of $82.4 \mathrm{mV} / \mathrm{s}$, at the output of the TIA, with an input referred dark current of $16.5 \mathrm{fA}$. Considering the pixel area of $3.84 \times 10^{-4} \mathrm{~cm}^{2}$, this equates to a dark current intensity of $0.043 \mathrm{nA} / \mathrm{cm}^{2}$.

The measured sensitivity of the $n$-well/p-subphotodiode is shown in Figure 11(b). The peak responsivity wavelength was between $700 \mathrm{~nm}$ and $800 \mathrm{~nm}$. More than 50 pulse width samples were collected at a particular optical power and measured by the power meter. Their mean and standard deviation were computed and plotted in Figure 11(c), where SNR is defined as their mean divided by their standard deviation. Figure 12 provides the relationship of the measured photocurrent and incident power, in which the photocurrent is calculated by measuring the pulse width and deduced based on (4) and (5).

4.3. p-Nitrophenol Detection. In order to do system-level test, a system measurement environment illustrated in Figure 13 is introduced instead of a fixed system box, because exciting board and sensor board will be changed during test to realize different input intensity and multisensor stability test, respectively. 


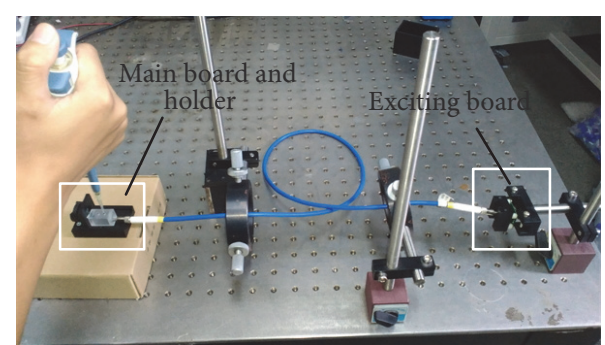

FIGURE 13: System measurement environment.

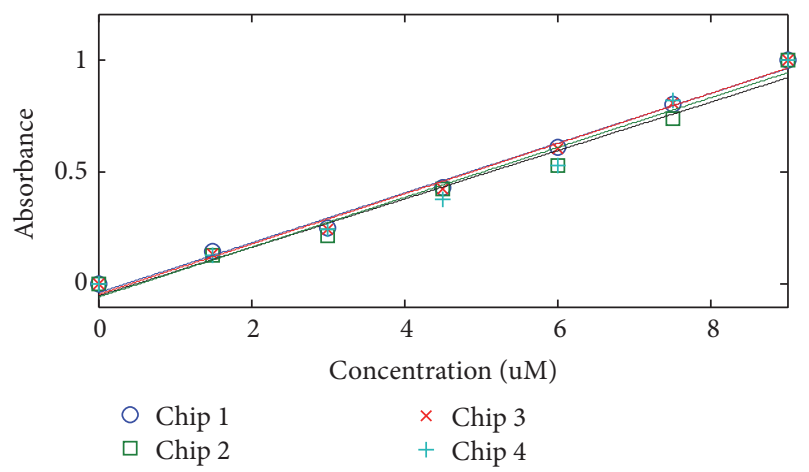

FIgURE 14: p-Nitrophenol detection with different photodetection sensor $\left(1 \times 10^{-6} \mathrm{~mol} / \mathrm{L}=1 \mu \mathrm{M}\right)$.

In the test, p-nitrophenol, which is the hydrolysis product of methyl parathion, is used as reagent during the measurements for the system stability, which includes multisensors stability test, different input light intensity stability test, and long term stability test.

The detection sensor consistency check and different input light intensity stability test are shown in Figures 14 and 15 , respectively. Four random photodetection sensor samples were used to measure six different concentrations of $\mathrm{p}$ nitrophenol from 0 to $9 \times 10^{-6} \mathrm{~mol} / \mathrm{L}$. The linear relationship of the results based on four different photodetection sensors is all above 0.97 and the LOD is below $0.20 \times 10^{-6} \mathrm{~mol} / \mathrm{L}$. As for the absorbance at 0.5 , the concentrations estimated from the linear relation from Figure 14 range from $0.4841 \times 10^{-6} \mathrm{~mol} / \mathrm{L}$ to $0.5018 \times 10^{-6} \mathrm{~mol} / \mathrm{L}$, which shows very little difference, illustrated in Table 1. Meanwhile, the linear relationship of the measurement results with different input light intensity is all above 0.99 as shown in Table 2. The difference between the estimated concentrations from the Figure 15 at absorbance 0.6 ranges from 0.6041 to 0.6641 . It can be inferred that the deviations of detection results based on different proposed sensors can be neglected and the variations in a range of the intensity of the exciting optical power also have enough low impact on the detection. Those will enable detection solution with the proposed sensor for disposable applications in future.

4.4. Methyl Parathion Detection. The methyl parathion hydrolase $(m p d)$ cloned from Stenotrophomonas sp. was got by traditional biological engineering methods and its

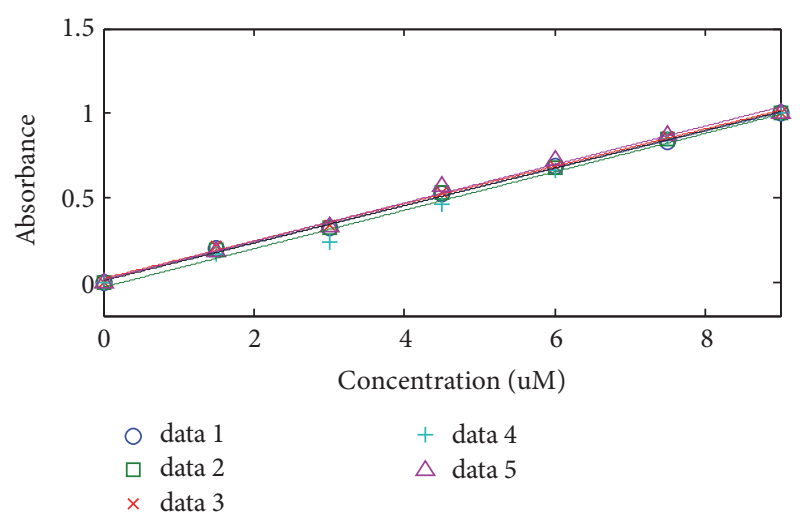

FIGURE 15: p-Nitrophenol detection with different input light intensity $\left(1 \times 10^{-6} \mathrm{~mol} / \mathrm{L}=1 \mu \mathrm{M}\right)$.

TABLE 1: Character of p-nitrophenol detection with different photodetection sensor.

\begin{tabular}{lcc}
\hline Identifier & Linear relationship & $\begin{array}{c}\text { Analytical conc. } \\
\left(10^{-6} \mathrm{~mol} / \mathrm{L}\right)\end{array}$ \\
\hline Chip 1 & 0.9924 & 0.4841 \\
Chip 2 & 0.9797 & 0.5113 \\
Chip 3 & 0.9919 & 0.4869 \\
Chip 4 & 0.9762 & 0.5018 \\
\hline
\end{tabular}

TABLE 2: Character of p-nitrophenol detection with different input light intensity.

\begin{tabular}{lcc}
\hline Input light intensity & $\begin{array}{c}\text { Linear } \\
\text { relationship }\end{array}$ & $\begin{array}{c}\text { Analytical conc. } \\
\left(10^{-6} \mathrm{~mol} / \mathrm{L}\right)\end{array}$ \\
\hline Data $1\left(1.6 \mu \mathrm{W} / \mathrm{cm}^{2}\right)$ & 0.9985 & 0.6641 \\
Data 2 $\left(1.3 \mu \mathrm{W} / \mathrm{cm}^{2}\right)$ & 0.9987 & 0.6227 \\
Data $3\left(0.9 \mu \mathrm{W} / \mathrm{cm}^{2}\right)$ & 0.9974 & 0.6135 \\
Data $4\left(0.7 \mu \mathrm{W} / \mathrm{cm}^{2}\right)$ & 0.9903 & 0.6423 \\
Data $5\left(0.4 \mu \mathrm{W} / \mathrm{cm}^{2}\right)$ & 0.9939 & 0.6041 \\
\hline
\end{tabular}

enzyme activity has been studied in detail in the literature [26-28, 30]. The enzyme can hydrolyze the p-nitrophenyl organophosphate compounds into yellow p-nitrophenolate ions and be applied in biosensing system for detection of organophosphate compounds. In this design, the organ phosphorus hydrolase $(\mathrm{OPH})$ is immobilized on the inner surface of the solution holder by the method of physical adsorption. In this utilized adsorption method, the enzyme solution is injected into the holder to incubate for enough time followed by pouring out the enzyme from the holder solution. This method can maintain good enzymatic activity due to no chemical modification on the enzyme molecules and is very easy to perform. One possible problem of this adsorption method is that the enzyme is absorbed loosely on the inner wall of the holder and is easier to leak. However, thorough experiments discover that the quality 


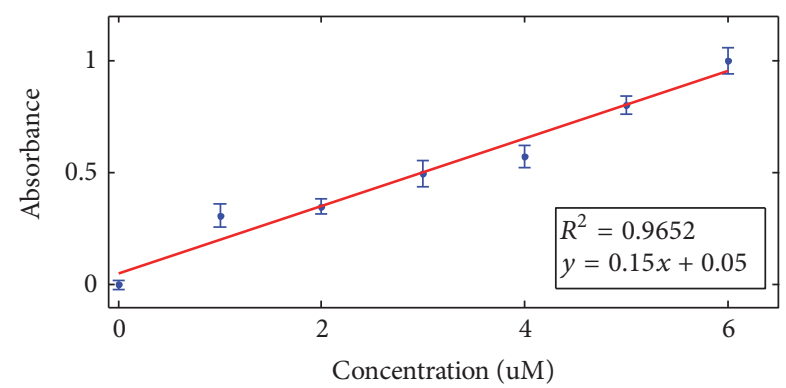

FIGURE 16: Methyl parathion detection in the proposed system.

TABLE 3: Comparison of the limit of detection of OP compounds.

\begin{tabular}{lccc}
\hline Parameter & This work & $\begin{array}{c}\text { [1] Sensors } \\
(2012)\end{array}$ & $\begin{array}{c}\text { [29] Sensors and } \\
\text { Actuators }(2015)\end{array}$ \\
\hline $\begin{array}{l}\text { Detection } \\
\text { method }\end{array}$ & $\begin{array}{c}\text { Optical } \\
\text { absorption } \\
(@ 405 \mathrm{~nm}) \\
\text { Mobile-based } \\
\text { Key } \\
\text { technologies }\end{array}$ & $\begin{array}{c}\text { Optical } \\
\text { absorption } \\
(@ 400 \mathrm{~nm})\end{array}$ & $\begin{array}{c}\text { Optical } \\
\text { absorption } \\
(@ 400 \mathrm{~nm})\end{array}$ \\
$\begin{array}{l}\text { Chotodiode } \\
\text { LOD }\end{array}$ & PIN-FET & $\begin{array}{c}\text { Spectrometer } \\
\text { with CCD }\end{array}$ \\
\hline
\end{tabular}

of this physical adsorption method could be guaranteed in a week at least by operating incubation for enough long duration. Therefore, the thin enzyme film can be prepared beforehand and then stored under low temperature before use, which will simplify the processes of on-field detection definitely.

To verify its effectiveness for OP compound detection, methyl parathion (one type of OP compound) is used as origins under detection. The used methyl parathion concentration values were designed in a range of 0 to $6 \times 10^{-6} \mathrm{~mol} / \mathrm{L}$ with 7 levels and the time for analysis ranged from one minute for the first sample to several minutes for the last sample. Test result illustrated in Figure 16 shows the significant linear relationship up to $6 \times 10^{-6} \mathrm{~mol} / \mathrm{L}\left(R^{2}=0.9652\right)$. The limit of detection (LOD) is estimated at $0.4 \times 10^{-6} \mathrm{~mol} / \mathrm{L}$.

The main characteristics of this work versus those published recently are given in Table 3 . In this design, the electronic system for detection is mainly based on customized integrated chip in which the electronic modules are integrated into one chip by very large scale integration (VLSI) methods, thus reducing the noise from the electronics and the interference coupled into the sensing chain, improving the sensitivity with smaller value of LOD, comparing with [1]. The method reported in [29] can get the lowest value of LOD reported so far for paraoxon detection using optical absorbance spectrometry or chemical colorimetry. However, since its colorimetric analytical method is based on the reduction of p-nitrophenyl substitute compounds by sodium borohydride accompanied with thermal enhancement up to almost $80^{\circ} \mathrm{C}$, the progress of analysis needs at least 40 minutes, which is too slow for the on-field application. Furthermore, its requirement for poisonous and dangerous chemical material $\left(\mathrm{NaBH}_{4}\right)$ during the analysis challenges the management/control for poisonous materials. As a result, although the value of LOD achieved in this work is a little higher than that of [29], the analysis progress seems very simple and does not require any dangerous chemical material to bring on-field.

\section{Conclusion}

The proposed photodetection sensor incorporates pulse width modulation with a folded reference, achieving a large dynamic range, high sensitivity, and low power consumption. Based on this photodetection sensor, a mobile-based high sensitivity absorptiometer is presented to detect organophosphorus (OP) compounds for Internet-of-Things based food safety tracking. The system is suitably applied in the field environment with the following reasons. Firstly, the enzyme is immobilized in the recycled holder in advance. It is very convenient for use because only sample under detection needs to be added to the holder. Secondly, the absorptiometer is rather compact and consumes low power with the supply of a coin battery. Meanwhile, its detection flow is integrated in the embedded code and android-based APP, while the detection results and intermediate raw data could be pushed into the cloud for record through a mobile, which further simplifies the operation during the on-field detection. In the end, it should be noted that the absorbance of OP will be affected by temperature variations and it can be addressed with calibration.

\section{Competing Interests}

The authors declare that there is no conflict of interests regarding the publication of this article.

\section{Acknowledgments}

The authors are grateful to NSFC program under Grant 61177021 for funding this work.

\section{References}

[1] W. Lan, G. Chen, F. Cui, F. Tan, R. Liu, and M. Yushupujiang, "Development of a novel optical biosensor for detection of organophoshorus pesticides based on methyl parathion hydrolase immobilized by metal-chelate affinity," Sensors, vol. 12, no. 7, pp. 8477-8490, 2012.

[2] J. M. Molina-Ruiz, E. Cieslik, and I. Walkowska, "Optimization of the QuEChERS method for determination of pesticide residues in chicken liver samples by gas chromatography-mass spectrometry," Food Analytical Methods, vol. 8, no. 4, pp. 898906, 2015.

[3] I. Montesinos, A. Sfakianaki, M. Gallego, and C. D. Stalikas, "Graphene-coated cotton fibers as a sorbent for the extraction of multiclass pesticide residues from water and their determination by gas chromatography with mass spectrometry," Journal of Separation Science, vol. 38, no. 5, pp. 836-843, 2015. 
[4] J. Nan, J. Wang, X. Piao et al., "Novel and rapid method for determination of organophosphorus pesticide residues in edible fungus using direct gas purge microsyringe extraction coupled on-line with gas chromatography-mass spectrometry," Talanta, vol. 142, pp. 64-71, 2015.

[5] R. Su, X. Xu, X. Wang et al., "Determination of organophosphorus pesticides in peanut oil by dispersive solid phase extraction gas chromatography-mass spectrometry," Journal of Chromatography B, vol. 879, no. 30, pp. 3423-3428, 2011.

[6] L. Araujo, M. E. Troconis, D. Cubillán, J. Mercado, N. Villa, and A. Prieto, "Single drop microextraction and gas chromatography-mass spectrometry for the determination of diflufenican, mepanipyrim, fipronil, and pretilachlor in water samples," Environmental Monitoring and Assessment, vol. 185, no. 12, pp. 10225-10233, 2013.

[7] K. Y. Ko, J. Y. Shin, D.-G. Kim, M. Kim, and S.-W. Son, "Determination of organophosphorus pesticides in stomach contents of postmortem animals by QuEChERS and gas chromatography," Journal of Analytical Toxicology, vol. 38, no. 9, pp. 667-671, 2014.

[8] M. A. Farajzadeh, M. R. A. Mogaddam, and H. Ghorbanpour, "Development of a new microextraction method based on elevated temperature dispersive liquid-liquid microextraction for determination of triazole pesticides residues in honey by gas chromatography-nitrogen phosphorus detection," Journal of Chromatography A, vol. 1347, pp. 8-16, 2014.

[9] C. Ruan, X. Zhao, and C. Liu, "Determination of diflubenzuron and chlorbenzuron in fruits by combining acetonitrile-based extraction with dispersive liquid-liquid microextraction followed by high-performance liquid chromatography," Journal of Separation Science, vol. 38, no. 17, pp. 2931-2937, 2015.

[10] N. Li, J. Chen, and Y.-P. Shi, "Magnetic graphene solid-phase extraction for the determination of carbamate pesticides in tomatoes coupled with high performance liquid chromatography," Talanta, vol. 141, pp. 212-219, 2015.

[11] C. Cortada, L. C. Dos Reis, L. Vidal, J. Llorca, and A. Canals, "Determination of cyclic and linear siloxanes in wastewater samples by ultrasound-assisted dispersive liquid-liquid microextraction followed by gas chromatography-mass spectrometry," Talanta, vol. 120, pp. 191-197, 2014.

[12] V. G. Amelin, D. S. Bol'Shakov, and A. V. Tretyakov, "Separation and quantification of polar pesticides in well, surface, and drinking water by capillary electrophoresis," Journal of Analytical Chemistry, vol. 67, no. 11, pp. 904-924, 2012.

[13] Y. Zhang, M. A. Arugula, M. Wales, J. Wild, and A. L. Simonian, "A novel layer-by-layer assembled multi-enzyme/CNT biosensor for discriminative detection between organophosphorus and non-organophosphrus pesticides," Biosensors and Bioelectronics, vol. 67, pp. 287-295, 2015.

[14] R. Khaksarinejad, A. Mohsenifar, T. Rahmani-Cherati, R. Karami, and M. Tabatabaei, "An Organophosphorus hydrolasebased biosensor for direct detection of paraoxon using silicacoated magnetic nanoparticles," Applied Biochemistry and Biotechnology, vol. 176, no. 2, pp. 359-371, 2015.

[15] A. Mulchandani, W. Chen, P. Mulchandani, J. Wang, and K. R. Rogers, "Biosensors for direct determination of organophosphate pesticides," Biosensors and Bioelectronics, vol. 16, no. 4-5, pp. 225-230, 2001.

[16] C. S. Pundir and N. Chauhan, "Acetylcholinesterase inhibitionbased biosensors for pesticide determination: a review," Analytical Biochemistry, vol. 429, no. 1, pp. 19-31, 2012.
[17] S. P. Sharma, L. N. S. Tomar, J. Acharya, A. Chaturvedi, M. V. S. Suryanarayan, and R. Jain, "Acetylcholinesterase inhibitionbased biosensor for amperometric detection of Sarin using single-walled carbon nanotube-modified ferrule graphite electrode," Sensors and Actuators B: Chemical, vol. 166-167, pp. 616623, 2012.

[18] V. Dhull, A. Gahlaut, N. Dilbaghi, and V. Hooda, "Acetylcholinesterase biosensors for electrochemical detection of organophosphorus compounds: a review," Biochemistry Research International, vol. 2013, Article ID 731501, 18 pages, 2013.

[19] J.-W. Choi, Y.-K. Kim, B.-K. Oh, S.-Y. Song, and W. H. Lee, "Optical biosensor for simultaneous detection of captan and organophosphorus compounds," Biosensors and Bioelectronics, vol. 18, no. 5-6, pp. 591-597, 2003.

[20] X. Sun, B. Liu, and K. Xia, "A sensitive and regenerable biosensor for organophosphate pesticide based on self-assembled multilayer film with CdTe as fluorescence probe," Luminescence, vol. 26, no. 6, pp. 616-621, 2011.

[21] http://www.ideaoptics.com/Products/PContent.aspx?pd= PG4004.

[22] Y. Chang, Y. Tai, Y. Huang, and Y. Yang, "A phototransistorbased high-sensitivity biosensing system using 650-nm light," IEEE Sensors Journal, vol. 9, no. 6, pp. 673-677, 2009.

[23] Y.-W. Chang, P.-C. Yu, Y.-T. Huang, and Y.-S. Yang, "A highsensitivity CMOS-compatible biosensing system based on absorption photometry," IEEE Sensors Journal, vol. 9, no. 2, pp. 120-127, 2009.

[24] B. Fowler, M. D. Godfrey, and S. Mims, "Reset noise reduction in capacitive sensors," IEEE Transactions on Circuits and Systems I: Regular Papers, vol. 53, no. 8, pp. 1658-1669, 2006.

[25] M. Kavruk, V. C. Özalp, and H. A. Öktem, "Portable bioactive paper-based sensor for quantification of pesticides," Journal of Analytical Methods in Chemistry, vol. 2013, Article ID 932946, 8 pages, 2013.

[26] J.-W. Choi, Y.-K. Kim, I.-H. Lee, J. Min, and W. H. Lee, "Optical organophosphorus biosensor consisting of acetylcholinesterase/viologen hetero Langmuir-Blodgett film," Biosensors and Bioelectronics, vol. 16, no. 9-12, pp. 937-943, 2001.

[27] G. A. Alonso, R. B. Dominguez, J.-L. Marty, and R. Muñoz, “An approach to an inhibition electronic tongue to detect on-line organophosphorus insecticides using a computer controlled multi-commuted flow system," Sensors, vol. 11, no. 4, pp. 3791$3802,2011$.

[28] L. Wan, Y. Qin, P. Chiang, G. Chen, R. Liu, and Z. Hong, "High-sensitivity photodetection sensor front-end, detecting organophosphourous compounds for food safety," in Proceedings of the IEEE Custom Integrated Circuits Conference (CICC '13), pp. 1-4, 2013.

[29] M.-P. N. Bui and A. Abbas, "Simple and rapid colorimetric detection of p-nitrophenyl substituent organophosphorous nerve agents," Sensors and Actuators B: Chemical, vol. 207, pp. 370-374, 2015.

[30] K. Murari, R. Etienne-Cummings, N. Thakor, and G. Cauwenberghs, "Which photodiode to use: a comparison of CMOScompatible structures," IEEE Sensors Journal, vol. 9, no. 7, pp. 752-760, 2009.

[31] K. Murari, R. Etienne-Cummings, N. V. Thakor, and G. Cauwenberghs, "A CMOS in-pixel CTIA high-sensitivity fluorescence imager," IEEE Transactions on Biomedical Circuits and Systems, vol. 5, no. 5, pp. 449-458, 2011.

[32] W. M. C. Sansen, Analog Design Essentials, Springer, Dordrecht, The Netherlands, 2006. 
[33] N. Liu, Z. Hong, and R. Liu, "A CMOS detector system for fluorescent bio-sensing application," in Proceedings of the IEEE Asian Solid-State Circuits Conference (A-SSCC '08), pp. 333-336, November 2008.

[34] C. Xu, S. Chao, and M. Chan, "A new correlated double sampling (CDS) technique for low voltage design environment in advanced CMOS technology," in Proceedings of the 28th European Solid-State Circuits Conference (ESSCIRC '02), pp. 117-120, IEEE, Florence, Italy, September 2002.

[35] C. Hu, P. Y. Chiang, K. Hu, H. Liu, R. Khanna, and J. Nejedlo, "A 90nm-CMOS, 500Mbps, fully-integrated IR-UWB transceiver using pulse injection-locking for receiver phase synchronization," in Proceedings of the IEEE Radio Frequency Integrated Circuits Symposium (RFIC '10), pp. 201-204, IEEE, Anaheim, Calif, USA, May 2010. 


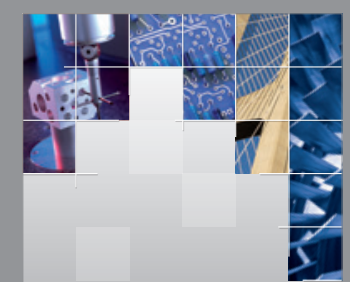

\section{Enfincering}
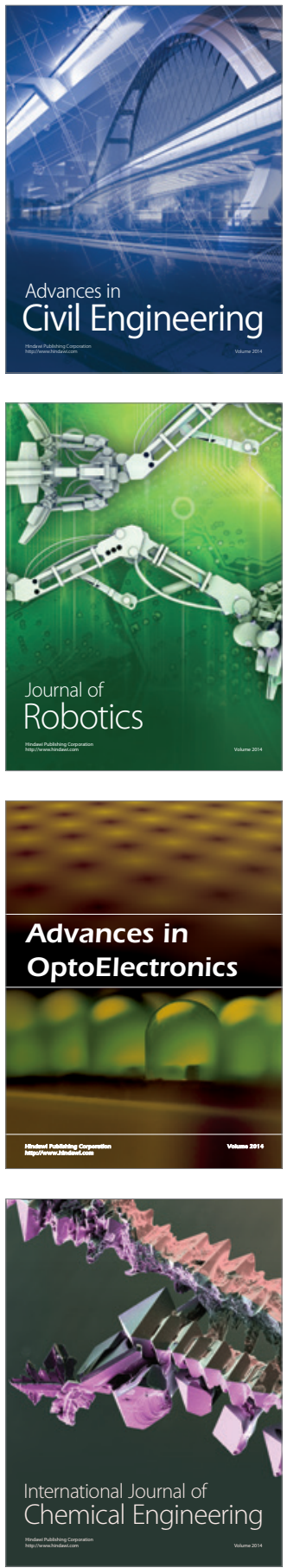

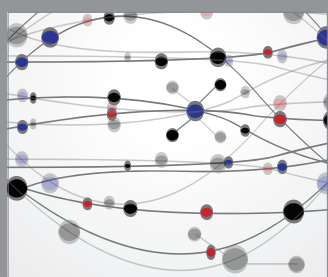

The Scientific World Journal

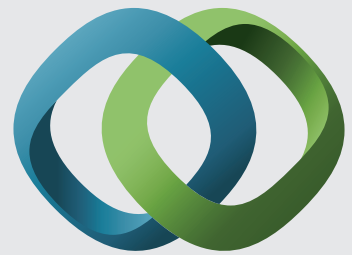

\section{Hindawi}

Submit your manuscripts at

https://www.hindawi.com
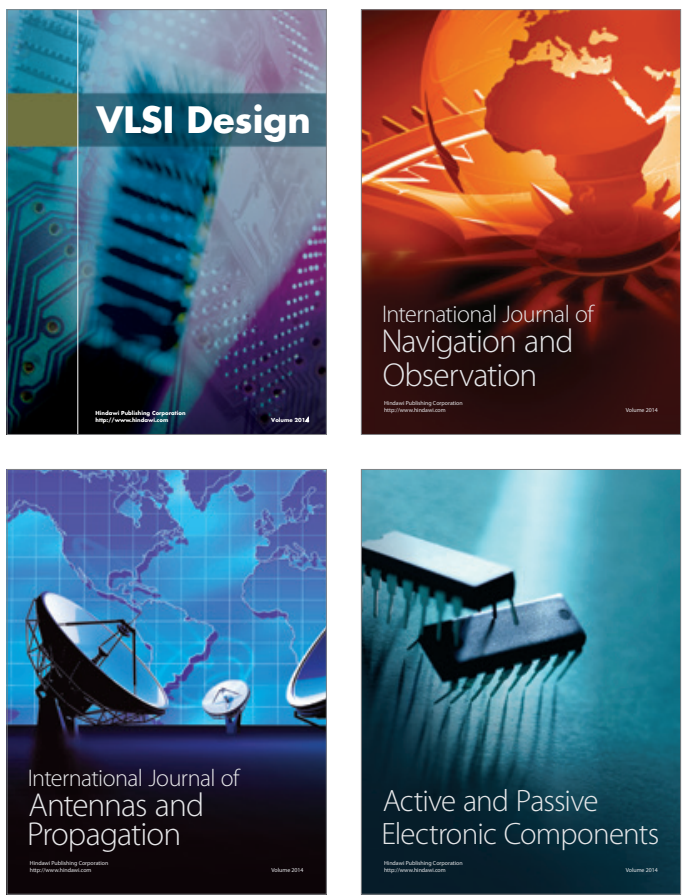
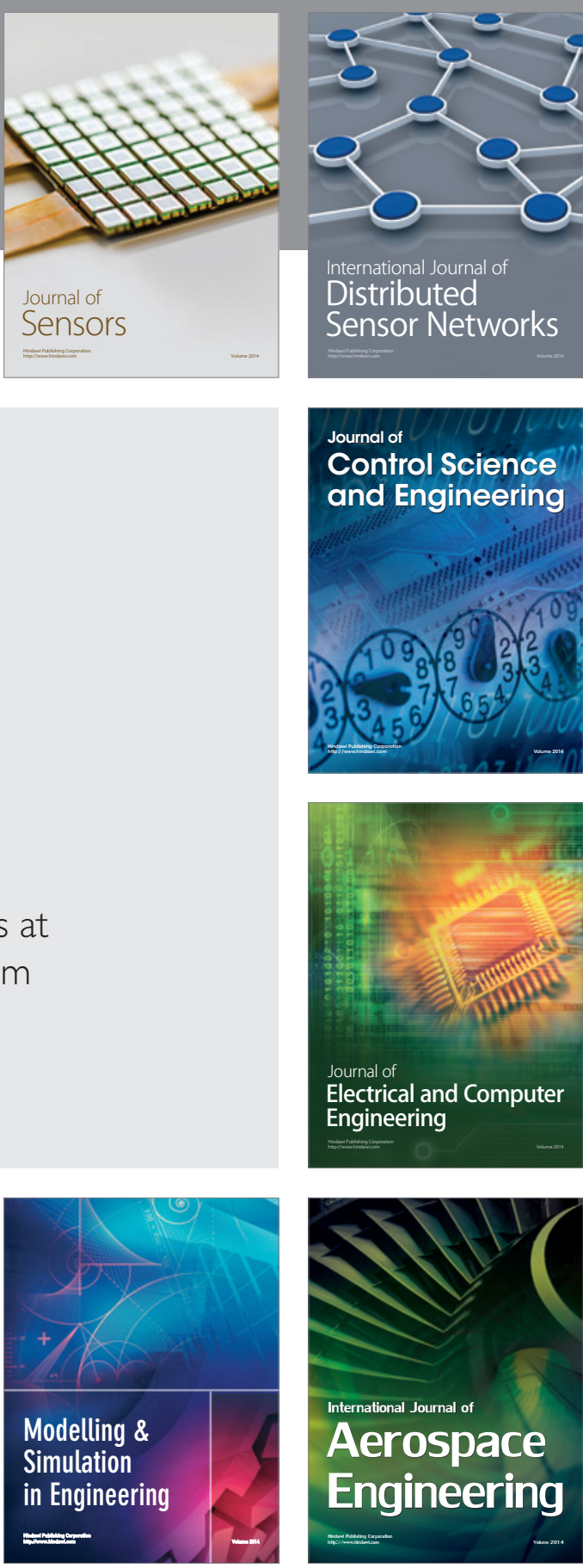

International Journal of

Distributed

Sensor Networks

$-$

Joumal of

Control Science

and Engineering
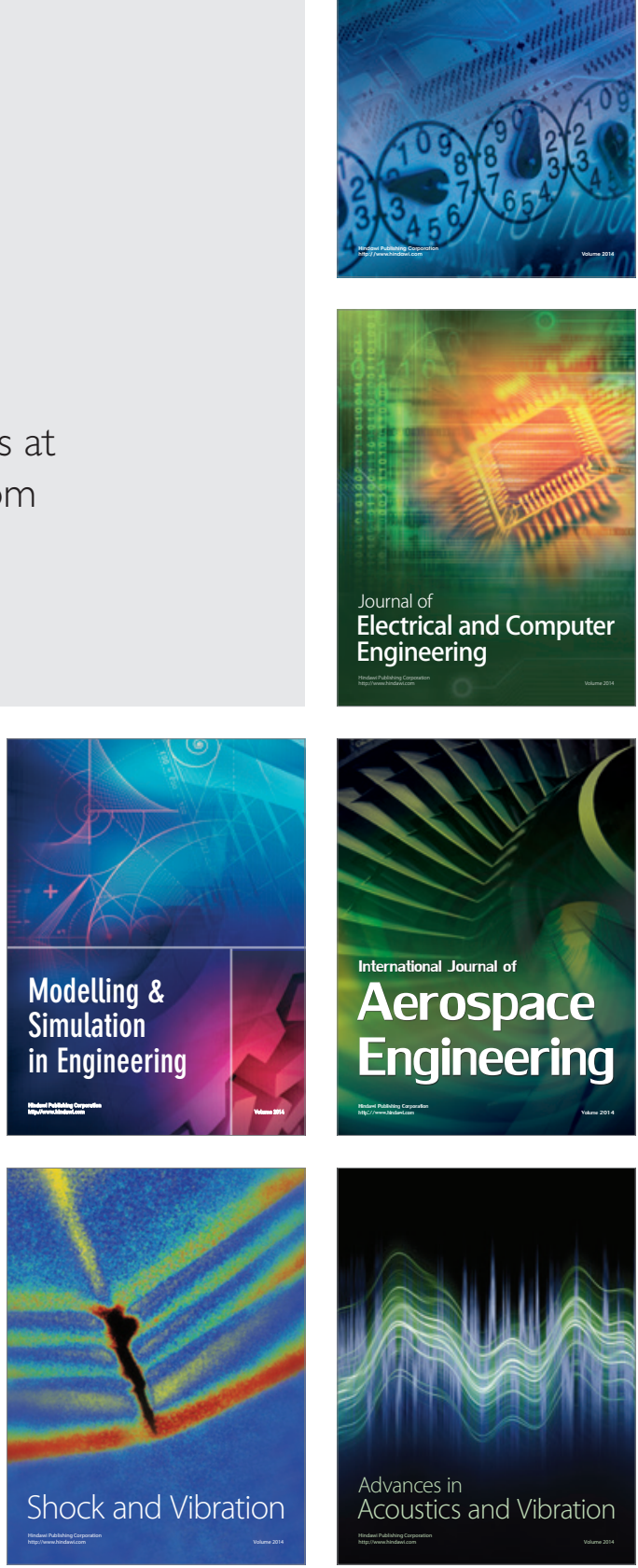